\title{
EFFECT OF FOLIAR APPLICATIONOF ANTI SALINITY SUBSTANCES ON GERANIUM (Pelargonium graveolens) PLANTS UNDER SALT STRESS
}

\author{
L. S. Gadallah ${ }^{1 *}$, M. A. EL-Mewafy El-Ghadban ${ }^{1}$, A. Sh. Soliman ${ }^{2}$, H. R.A. Mehasen ${ }^{1}$ and M. S. Abbas ${ }^{2}$ \\ ${ }^{1}$ Medicinal and Aromatic Plants Dept., Horticultural Research Institute (HRI), Agricultural Research Center (ARC), \\ Giza, Egypt. \\ ${ }^{2}$ Natural Resources Department, Faculty of African Postgraduate Studies, Cairo University, Egypt. \\ *Corresponding Author's email: samylamis@ymail.com
}

\begin{abstract}
Salinity limits crop metabolic activities, hampers plant growth and synthesis of secondary metabolites affecting the osmotic potential in the root zone of plants. The present investigation was carried out to improve the aromatic oil of geranium plants productivity using saline water at alternative irrigations with good quality water. The field experiment was carried out using saline water $\left(3.125,4.69,6.25 \mathrm{dSm}^{-1}\right.$ and tap water), moringa extract $\left(2.5,5\right.$ and $\left.10 \mathrm{gL}^{-1}\right)$, ascorbic acid $\left(0.1,0.2\right.$ and $\left.0.3 \mathrm{gL}^{-1}\right)$ and proline $\left(25,50\right.$ and $\left.100 \mathrm{mgL}^{-1}\right)$ as a foliar application. The obtained data illustrated that, the yields of essential oil per hectare were maximum when tap water combined with $10 \mathrm{gL}^{-1}$ of moringa extract. The results showed that Moring extract as foliar application at $10 \mathrm{~g} \mathrm{~L}^{-1}$ improved that the plant growth following $0.3 \mathrm{~g} \mathrm{~L}^{-1}$.It can be concluded that the utilization of moringa extracts as foliar spray to avoid the salt stress, following vitamin $\mathrm{C}$ protected well cells and elongated the roots. The present results are very useful for a farmer in the newly reclaimed areas who face the limited saline irrigation water resources because moringa extracts have a positive effect on vegetative growth and chemical contents of economic plant.
\end{abstract}

Key words: Geranium, water salinity, moringa extracts, ascorbic acid and proline. https://doi.org/10.36899/JAPS.2020.3.0085 Published online March 25, 2020

\section{INTRODUCTION}

Pelargonium graveolens (family Graniaceae) is grown for essential oil production in Egypt, China, Morocco, India and South Africa and many other countries (Wiess, 1997). The giranjol and linalool found ingenus Plargonium play an important on the synthesis of antioxidants that destroy cancer cells especially breast cancer and any infections within the body by bacteria and fungi (Wiess, 1997).

Salinity is an environmental condition that induces stress on plants especially when saline water is used irrigation. It limits crop metabolic activities, hampers plant growth and synthesis of secondary metabolites, by affecting osmotic potential in the root zone of plants (Baâtour et al., 2012). Salinity induces ion toxicity due to excessive uptake of sodium $\left(\mathrm{Na}^{+}\right)$and inhibits plant growth and development by increasing the osmotic stress and nutrient imbalance (Cornillon and Pallix, 1997; El-Baz et al., 2003). The extent of damage depends on the severity of stress, growth condition and plant sensitivity to salinity (Cornillon and Pallix, 1997).

It was found that, saline irrigation water affected most of the morphological (Eid et al., 2011; Caliskan et al. 2017) and vegetative growth parameters (plant height, number of branches, leaf length and shoots fresh weight) of medicinal plants (Maia et al., 2017). Under saline condition, the most problem facing agricultural production in irrigated arid and semi-arid areas is how to prepare a suitable root zone. Therefore, many investigators studied the effect of certain foliar application on the growth growing parameters under salinity conditions (Mazher et al., 2011; Abdalla, 2013, Soltani et al., 2014). They found that, the increasing concentration of foliar application such as ascorbic acid, moringa leaves extract gradually increased the growth parameters of medicinal plants.

In Egypt, saline water is used for irrigation under the arid climatic conditions in certain areas of Egypt with either imperfect drainage system, increasing of water-table level or the relatively high salinity levels of water sources particularly in the new reclaimed land, with the increasing need for salt tolerant medicinal and aromatic plants due to both increasing restriction of water resources and saline water intrusion into ground water especially in newly reclaimed lands. Therefore, the present study aimed to study the using of foliar applications of moringa extract, ascorbic acid and proline at different concentrations on the growth parameters, chemical composition and essential oil of geranium plant irrigated by saline water.

\section{MATERIALS AND METHODS}

Experimental layout: The current study was conducted to investigate the effect of salt stress for geranium (Pelargonium graveolens) as well as using some foliar applications to enhance the plant growth and productivity 
under sea salt conditions. The experiment was performed during 2016 and 2017 summer seasons (open field) at Technical of the Guiding Towards (TGT), Agricultural Research Center (ARC), Dokki, Giza, Egypt. The coordinate of the experimental farm was N30.04 and E31.20o. The maximum and minimum air temperatures during April until July 2016 ranged from 28.43-36.30 and 14.09-19.61 ${ }^{\circ} \mathrm{C}$, respectively, while the percentage relative humidity ranged from $42.93-48.05$. For 2017 , the maximum and minimum air temperatures during April until July ranged from $27.86-35.57$ and $14.37-20.19^{\circ} \mathrm{C}$, respectively, while the percentage relative humidity ranged from $36.20-44.21$

Experimental design: The experimental design was spilt plot with three replicates four levels of sea salt concentrations $\left(3.125,4.36,6.25 \mathrm{dSm}^{-1}\right.$ and tap water) was allocated in the main plot. The levels of saline irrigation water were prepared by using sea salt (Rasheedy salt). The soluble ions of this salt are $\mathrm{CO}_{3}^{--}$, $\mathrm{Cl}^{-}, \mathrm{SO}^{--}, \mathrm{Mg}^{++}, \mathrm{Na}^{+}$and $\mathrm{K}^{+}$at level $10,68.75,7.95,2.0$, $82.4,1.20$ and 2.30 meq. $\mathrm{L}^{-1}$, respectively.

Three levels of each foliar application (moringa extract, ascorbic acid and proline) were allocated in sub main plots. Three foliar applications by three concentrations moringa extract $\left(2.5,5\right.$ and $\left.10 \mathrm{gL}^{-1}\right)$, ascorbic acid $\left(0.1,0.2\right.$ and $\left.0.3 \mathrm{gL}^{-1}\right)$ and proline $(25,50$ and $100 \mathrm{mgL}^{-1}$ ).

$$
\begin{aligned}
& \text { essential oil } \%=\frac{\text { essential oil vol. (measuring pipette reading) }}{\text { weight of sample }} \times 100 \\
& \text { essential oil yield }(\mathrm{ml}) \text { per plant }=\text { oil percentage } \times \text { canopy fresh weight }(\mathrm{g}) \text { per plant }
\end{aligned}
$$

The air-dried canopy of each sample was hydro distilled in a Clevenger -type apparatus for $4 \mathrm{~h}$, according to the procedure described in the Egyptian pharmacopeia (2005) to determine the volatile oil percentage (Volume/Weight).

Gas chromatography (GC) analysis: The investigation of odorants is not an easy task, which needs to be undertaken in the context of fit-for-purpose quality systems. To date, great attention has been paid to determination of the volatile fractions of odorants, since they are responsible for the attributes of global flavor [i.e. a combination of olfactory (aroma) and gustatory (taste) sensations produced by chemicals]. This kind of determination can be carried out by analytical techniques [e.g., gas chromatography (GC) combined with mass spectrometry and/or olfactometric GC]. Methods complementary to $\mathrm{GC}$ analysis are available, allowing assessment of the olfactory impact by an electronic nose (e-nose) or a panel of selected individuals. Also, we consider some innovative analytical techniques to study the effects of odorants in food during consumption (Biniecka and Caroli, 2011)
Moringa extracts: Moringa extract was prepared by homogenized $40 \mathrm{~g}$ of dried powder with $100 \mathrm{ml}$ from tap water and left for $24 \mathrm{~h}$ in the dark at room temperature, stirring frequently with a glass rod. The extract was obtained by filtration (Whatman No.1).Filtrate was concentrated using Rotary Evaporator (Model RE52A, china),

The ten foliar applications: Moringa extracts $(2.5,5$ and $\left.10 \mathrm{~g} \mathrm{~L}^{-1}\right)$, ascorbic acid $\left(0.1,0.2\right.$ and $\left.0.3 \mathrm{~g} \mathrm{~L}^{-1}\right)$, proline acid $\left(25,50\right.$ and $\left.100 \mathrm{mg} \mathrm{L}^{-1}\right)$ and controls were tested.

Plant materials: Seedlings of geranium local variety were obtained from El-Salam Arboretum Subordinated which belongs to the Governmental Protected Cultivation Sector. Geranium seedlings were transplanted to black plastic pots (30 L) on 12 and 16 April 2016 and 2017.

Vegetative yield characters: Yield parameters (fresh weight and dry weight $\left(\mathrm{KgHectare}^{-1}\right)$.

\section{Chemical composition:}

- Essential oil \% in fresh canopy.

- Essential oil yield per plant.

- Essential oil percentage and essential oil yield in the herb of geranium:

- Oil percentage and essential oil yield per plant were estimated in fresh canopy of geranium in both seasons according to the following equations:
Statistical analysis: The experiment was laid out in split plot design with three replications, where saline irrigation water levels were distributed in the main plot and foliar applications allocated in sub-plots. Analysis of data was done Statistix- 8 , to calculate the analysis of variance (ANOVA). The differences among means for all traits were tested for significance at the $\%$ level according to Waller and Duncan (1969).

\section{RESULTS AND DISCUSSION}

Vegetative growth yield in geranium: There were significant differences among the studied treatments (Tables 1 and 2). Referring the salinity level of irrigation water, the highest value of vegetative characters (fresh weight per hectare and dry weight per hectare) were obtained from tap water treatment (without salinity). The lowest vegetative character was obtained by the highest level of salt of irrigation water $\left(6.25 \mathrm{dSm}^{-1}\right)$.

Regarding the foliar application treatments data revealed that the highest value of vegetative character was obtained by using Moringa extract $\left(10 \mathrm{~g} \mathrm{~L}^{-1}\right)$ compared to the other foliar application treatments. The 
lowest vegetative characters value was obtained by without foliar application (control). The same results were obtained during the second season.

The interaction effect between salt concentration and foliar application showed that the highest vegetative characters were obtained by the tap water treatment combined by moringa extract $\left(10 \mathrm{~g} \mathrm{~L}^{-1}\right)$. The lowest vegetative character was obtained from the highest salt concentration $\left(6.25 \mathrm{dSm}^{-1}\right)$ combined by control (without foliar application). The obtained results agreed with Maia et al. (2017) and Soltani et al. (2016) who reported using some foliar applications such as moringa extract, ascorbic acid and proline led to improving geranium plant growth and productivity under saline irrigation water treatments. The same authors added that increase salinity level led to decrease plant growth regardless of foliar application. One the other hands, Soltani et al. (2016) found that increasing ascorbic acid concentration led to reducing the negative impacts of salinity of plant growth. Also, Veras (2017) mentioned that foliar application by extract moringa leaves led to enhance growth parameters under high irrigation water salinity.

\section{Essential oil production:}

Essential oil percentage (\%): Data in Table (3) show essential oil percentage in fresh leaves of geranium. Salinity irrigation water levels treatments were significant differences among the studied treatments, the highest percentage of essential oil was found in irrigation water treatment by $6.25 \mathrm{dSm}^{-1}$ salinity treatment; while the lowest of essential oil percent was gained by tap water treatment. The same results were obtained during the second season.

Regarding the foliar application treatments, data indicated that the highest essential oil percent by using moringa extract $\left(10 \mathrm{gL}^{-1}\right)$ compared with other foliar applications followed by Ascorbic acid $\left(0.3 \mathrm{gL}^{-1}\right)$ foliar application

The interaction effect between salt concentration and foliar application show that the highest essential oil percent was preceded by using moringa extract $\left(10 \mathrm{gL}^{-1}\right)$ foliar application with salinity irrigation water $\left(6.25 \mathrm{dSm}^{-}\right.$ ${ }^{1}$ ) in comparison with control (without foliar application). These results are in agreement with Dadkhah (2010) and Jelali et al. (2010) on geranium, who indicated that the essential oil percentage was increased under height value of saline condition. On the same trend, Abdalla et al. (2013) and Zoran et al (2015) reported that foliar application (moringa extract) increased the percentage of essential oil and oil yield under saline irrigation water.

Essential Oil yield per Hectare: Data in Table (4) presented that essential oil yield per hectare. Salinity levels treatments had significant differences among treatments. The highest value of essential oil yield per hectare was obtained when tap water treatment was used, while the lowest yield was obtained when higher concentration of saline water was used $\left(6.25 \mathrm{dSm}^{-1}\right)$. The same trend was obtained during the second season.

Regarding the foliar application treatments, data revealed that the highest essential oil yield per hectare was obtained when moringa extract at level of $10 \mathrm{gL}^{-1}$ was used

The interaction effect among salt concentration and foliar application showed that the highest total essential oil percentage was found by using moringa extract at level of $10 \mathrm{gL}^{-1}$ compared with control and ascorbic acid and proline treatments. The main essential oils percentage of geranium increased with increasing the salinity level regardless of foliar application treatments; while the total main essential oils per hectare increased with decreasing salinity level due to the high vegetative growth for the low salinity treatments. It was found that, foliar spray of moringa leaves extract led to increasing dry weight, carotenoid contents free proline, ascorbic acid, phenol, total sugars, total protein, total phenols and contents of N, P, K and Ca (Abdalla, 2013). These results were in line with obtained by Dadkhah (2010), Aziz et al. (2008) and Jelali et al. (2010), where the increased salinity levels led to decrease the essential oil percentage and total essential oil yield per hectare. Also, the present results are in harmony with Abdalla (2013) and Veras (2017) where the usage of the moringa leaves extract was significantly improved the total essential oil yield per plant.

Essential oil constituent: Data in Table (5) presented the composition of essential geranium oil. The main constituents of geranium oil were geraniol, eugenol, and myrcene. In addition, data showed that the highest values of meraniol observed with6.25 $\mathrm{dSm}^{-1}$ salinity irrigation water treatment with foliar application moringa extract $\left(10 \mathrm{gL}^{-1}\right)$ treatment. On the other hand, the highest values of eugenol observed with $6.25 \mathrm{dSm}^{-1}$ salinity irrigation treatment combined by foliar application moringa extract $\left(10 \mathrm{gL}^{-1}\right)$ treatment. Moreover, the highest values of linalool observed with $4.69 \mathrm{dSm}^{-1}$ salinity irrigation treatment with foliar application of moringa extract $\left(10 \mathrm{gL}^{-1}\right)$; the lowest values recorded by $6.25 \mathrm{dSm}^{-1}$ salinity irrigation treatment with foliar application of proline concentration $\left(25 \mathrm{mgL}^{-1}\right)$.

Andre et al. (2008) and Zoran et al. (2015) mentioned that the essential geranium oil components were myrcene, meraniol, eugenol, linalool, geranioland among others. 
Table 1. Effect of foliar application on fresh weight per Hectare(Kg) of geranium under salt stress during seasons 2016 and 2017.

\begin{tabular}{|c|c|c|c|c|c|c|c|c|c|c|c|}
\hline \multirow{2}{*}{$\begin{array}{r}\text { Foliar } \\
\text { applications }\end{array}$} & \multicolumn{2}{|c|}{ Moringa extracts $\left(\mathrm{gL}^{-1}\right)$} & \multicolumn{4}{|c|}{ Ascorbic acid(gL $\left.{ }^{-1}\right)$} & \multicolumn{5}{|c|}{ Proline acid $\left(\mathrm{mgL}^{-1}\right)$} \\
\hline & 2.5 & 5 & 10 & 0.1 & 0.2 & 0.3 & 25 & 50 & 100 & Control & Mean A \\
\hline Salinity & \multicolumn{11}{|c|}{ First season } \\
\hline Tap & $6534.2^{\mathrm{MNO}}$ & $7463.0^{\mathrm{EF}}$ & $9000.9^{\mathrm{A}}$ & $6654.7^{\mathrm{K}-\mathrm{N}}$ & $6806.9^{\mathrm{JKL}}$ & $7351.7^{\mathrm{EFG}}$ & $6943.2^{\mathrm{IJ}}$ & $7040.0^{\mathrm{C}}$ & $7163.2^{\mathrm{DE}}$ & $6742.6^{\mathrm{J}-\mathrm{M}}$ & $6870.0^{\mathrm{A}}$ \\
\hline $3.125 \mathrm{dsm}^{-1}$ & $6462.7^{\mathrm{NO}}$ & $7223.0^{\mathrm{FGH}}$ & $6534.2^{\mathrm{MNO}}$ & $6558.7^{\mathrm{LO}}$ & $6438.7^{\mathrm{NOP}}$ & $6823.20^{\mathrm{JK}}$ & $5925.6^{\mathrm{RS}}$ & $6982.5^{\mathrm{HIJ}}$ & $7847.5^{\mathrm{CD}}$ & $5934.2^{\mathrm{RS}}$ & $6643.0^{\mathrm{B}}$ \\
\hline $4.69 \mathrm{dsm}^{-1}$ & $6208.2^{\mathrm{F}-\mathrm{I}}$ & $6095.4^{\mathrm{GHI}}$ & $6351.6^{\mathrm{EFG}}$ & $6294.7^{\mathrm{OPQ}}$ & $5573.7^{\mathrm{T}}$ & $6175.7^{\mathrm{PQR}}$ & $6102.2^{\mathrm{QRS}}$ & $5429.7^{\mathrm{T}}$ & $5958.2^{\mathrm{RS}}$ & $5909.7^{\mathrm{S}}$ & $6109.9^{\mathrm{C}}$ \\
\hline $6.25 \mathrm{dsm}^{-1}$ & $2956.8^{\mathrm{VW}}$ & $2700.9^{\mathrm{wx}}$ & $3532.3^{\mathrm{U}}$ & $2995.2^{\mathrm{V}}$ & $3060.0^{\mathrm{V}}$ & $3573.6^{\mathrm{U}}$ & $3006.2^{\mathrm{V}}$ & $3187.7^{\mathrm{V}}$ & $2658.7^{\mathrm{X}}$ & $2482.6^{\mathrm{X}}$ & $3015.4^{\mathrm{D}}$ \\
\hline Mean B & $5540.5^{\mathrm{D}}$ & $5870.6^{\mathrm{B}}$ & $6354.8^{\mathrm{A}}$ & $5625.8^{\mathrm{E}}$ & $5469.8^{\mathrm{F}}$ & $5981.1^{\mathrm{B}}$ & $5494.3^{\mathrm{F}}$ & $5659.9^{\mathrm{CD}}$ & $5906.9^{\mathrm{BC}}$ & $5267.3^{\mathrm{G}}$ & \\
\hline Salinity & \multicolumn{11}{|c|}{ Second season } \\
\hline Tap & $6750.7^{\mathrm{J}}$ & $7507.7^{\mathrm{FG}}$ & $9165.1^{\mathrm{A}}$ & $6703.0^{\mathrm{FG}}$ & $6828.6^{\mathrm{B}}$ & $7570.9^{\mathrm{B}}$ & $6958.6^{\mathrm{B}}$ & $7372.5^{\mathrm{CD}}$ & $7192.0^{\mathrm{EF}}$ & $6790.8^{\mathrm{IJ}}$ & $7283.9^{\mathrm{A}}$ \\
\hline $3.125 \mathrm{dSm}^{-1}$ & $6656.2^{\mathrm{J}}$ & $7327.7^{\mathrm{H}}$ & $6783.7^{\mathrm{FG}}$ & $6654.0^{\mathrm{J}}$ & $6255.2^{\mathrm{HI}}$ & $6615.2^{\mathrm{G}}$ & $6054.2^{\mathrm{K}}$ & $6985.6^{\mathrm{DE}}$ & $7876.0^{\mathrm{E}}$ & $6126.7^{\mathrm{J}}$ & $6933.5^{\mathrm{B}}$ \\
\hline $4.69 \mathrm{dSm}^{-1}$ & $6280.2^{\mathrm{EF}}$ & $6115.2^{\mathrm{IJ}}$ & $6381.7^{\mathrm{FG}}$ & $6312.5^{\mathrm{BC}}$ & $6328.0^{\mathrm{BC}}$ & $6199.5^{\mathrm{FG}}$ & $6137.1^{\mathrm{B}}$ & $6214.1^{\mathrm{K}}$ & $6342.7^{\mathrm{K}}$ & $6182.4^{\mathrm{K}}$ & $6249.3^{\mathrm{C}}$ \\
\hline $6.25 \mathrm{dSm}^{-1}$ & $5365.7^{\mathrm{OP}}$ & $5902.6^{\mathrm{MNO}}$ & $6077.5^{\mathrm{MN}}$ & $5058.0^{\mathrm{P}}$ & $3732.0^{\mathrm{LM}}$ & $3794.8^{\mathrm{L}}$ & $3251.5^{\mathrm{NOP}}$ & $4004.2^{\mathrm{L}}$ & $2706.7^{Q}$ & $2675.0^{\mathrm{Q}}$ & $3956 \mathrm{~s} .8^{\mathrm{D}}$ \\
\hline Mean B & $6263.2^{\mathrm{E}}$ & $6713.3^{\mathrm{D}}$ & $7102.0^{\mathrm{A}}$ & $6594.2^{\mathrm{C}}$ & $5760.9^{\mathrm{F}}$ & $6045.1^{\mathrm{B}}$ & $5600.4^{\mathrm{B}}$ & $6144.1^{\mathrm{BC}}$ & $6029.4^{\mathrm{DE}}$ & $5443.7^{\mathrm{F}}$ & \\
\hline
\end{tabular}

Means within the letter and having the letters are not significantly from each other at significantly level of 0.05

Table (2): Effect of foliar application on dry weight per Hectare (Kg) of geranium under salt stress during seasons 2016 and 2017.

\begin{tabular}{|c|c|c|c|c|c|c|c|c|c|c|c|}
\hline \multirow{2}{*}{$\begin{array}{r}\text { Foliar } \\
\text { application }\end{array}$} & \multicolumn{3}{|c|}{ Moringa extracts $\left(\mathrm{gL}^{-1}\right)$} & \multicolumn{2}{|c|}{ Ascorbic acid $\left(\mathrm{gL}^{-1}\right)$} & \multicolumn{3}{|c|}{ Proline acid $\left(\mathrm{mgL}^{-1}\right)$} & \multirow[b]{2}{*}{100} & \multirow[b]{2}{*}{ Control } & \multirow[b]{2}{*}{ Mean A } \\
\hline & 2.5 & 5 & $\mathbf{1 0}$ & 0.1 & 0.2 & 0.3 & 25 & 50 & & & \\
\hline Salinity & \multicolumn{11}{|c|}{ First season 2016} \\
\hline Tap & $1187.0^{\mathrm{N}-\mathrm{Q}}$ & $1855.2^{\mathrm{B}-\mathrm{E}}$ & $2195.0^{\mathrm{A}}$ & $1627.8^{\mathrm{G}-\mathrm{M}}$ & $1697.9^{\mathrm{F}-\mathrm{L}}$ & $1915.2^{\mathrm{BCD}}$ & $1551.2^{\text {E-I }}$ & $1552.6^{\mathrm{E}-\mathrm{I}}$ & $1535.2^{\mathrm{C}-\mathrm{H}}$ & $1398.2^{\mathrm{J}-\mathrm{O}}$ & $1651.5^{\mathrm{A}}$ \\
\hline $3.125 \mathrm{dsm}^{-1}$ & $1149.8^{\mathrm{AB}}$ & $1638.1^{\mathrm{I}-\mathrm{N}}$ & $1832.6^{\mathrm{B}-\mathrm{F}}$ & $1509.4^{\mathrm{D}-\mathrm{I}}$ & $1523.7^{\mathrm{C}-\mathrm{H}}$ & $1733.7^{\mathrm{AB}}$ & $1188.0^{\mathrm{N}-\mathrm{Q}}$ & $1346.4^{\mathrm{L}-\mathrm{P}}$ & $1506.7^{\mathrm{G}-\mathrm{M}}$ & $1067.0^{\mathrm{Q}}$ & $1449.5^{\mathrm{B}}$ \\
\hline $4.69 \mathrm{dsm}^{-1}$ & $1147.4^{\mathrm{F}-\mathrm{L}}$ & $1629.0^{\mathrm{D}-\mathrm{I}}$ & $1657.9^{\mathrm{E}-\mathrm{I}}$ & $1342.2^{\mathrm{M}-\mathrm{Q}}$ & $1505.8^{\mathrm{E}-\mathrm{K}}$ & $1679.0^{\mathrm{D}-\mathrm{I}}$ & $1154.4^{\mathrm{OPQ}}$ & $1188.0^{\mathrm{N}-\mathrm{Q}}$ & $1347.4^{\mathrm{E}-\mathrm{J}}$ & $1154.4^{\mathrm{OPQ}}$ & $1380.5^{\mathrm{B}}$ \\
\hline $6.25 \mathrm{dsm}^{-1}$ & $1027.8^{\mathrm{G}-\mathrm{M}}$ & $1607.2^{\mathrm{C}-\mathrm{G}}$ & $1559.5^{\mathrm{G}-\mathrm{L}}$ & $1304.5^{\mathrm{L}-\mathrm{P}}$ & $1377.6^{\mathrm{K}-\mathrm{Q}}$ & $1499.5^{\mathrm{H}-\mathrm{M}}$ & $1219.2^{\mathrm{N}-\mathrm{Q}}$ & $1350.2^{\mathrm{L}-\mathrm{P}}$ & $1388.6^{\mathrm{K}-\mathrm{Q}}$ & $1311.8^{\mathrm{PQ}}$ & $1364.6^{\mathrm{B}}$ \\
\hline Mean B & $1128.0^{\mathrm{B}}$ & $1682.4^{\mathrm{AB}}$ & $1811.3^{\mathrm{A}}$ & $1445.9^{\mathrm{CD}}$ & $1526.3^{\mathrm{BC}}$ & $1706.9^{\mathrm{B}}$ & $1278.2^{\mathrm{EF}}$ & $1359.3^{\mathrm{DE}}$ & $1444.5^{\mathrm{BC}}$ & $1232.9^{\mathrm{F}}$ & \\
\hline Salinity & \multicolumn{11}{|c|}{ Second season 2017} \\
\hline Tap & $1243.2^{\mathrm{PQ}}$ & $1640.2^{\mathrm{I}-\mathrm{M}}$ & $2318.4^{\mathrm{A}}$ & $1648.9^{\mathrm{J}-\mathrm{P}}$ & $1698.7^{\mathrm{IJK}}$ & $1870.2^{\mathrm{EF}}$ & $1556.4^{\mathrm{GHI}}$ & $1557.9^{\mathrm{HI}}$ & $1538.3^{\mathrm{IJ}}$ & $1403.2^{\mathrm{K}-\mathrm{P}}$ & $1647.5^{\mathrm{A}}$ \\
\hline $3.125 \mathrm{dsm}^{-1}$ & $1381.4^{\mathrm{D}}$ & $1657.4^{\mathrm{BC}}$ & $1836.4^{\mathrm{EF}}$ & $1836.0^{\mathrm{EFG}}$ & $1811.5^{\mathrm{D}}$ & $1588.8^{\mathrm{CD}}$ & $1574.4^{\mathrm{B}}$ & $1580.6^{\mathrm{D}}$ & $1329.1^{\mathrm{FGH}}$ & $1223.0^{\mathrm{I}-\mathrm{L}}$ & $1581.9^{\mathrm{B}}$ \\
\hline $4.69 \mathrm{dsm}^{-1}$ & $1238.4^{\mathrm{PQ}}$ & $1541.8^{\mathrm{I}-\mathrm{O}}$ & $1593.6^{\mathrm{FGH}}$ & $1564.8^{\mathrm{I}-\mathrm{N}}$ & $1589.3^{\mathrm{I}-\mathrm{M}}$ & $1478.4^{\mathrm{JP}}$ & $1270.1^{\mathrm{OPQ}}$ & $1358.8^{\mathrm{M}-\mathrm{Q}}$ & $1291.2^{\mathrm{N}-\mathrm{Q}}$ & $1251.3^{\mathrm{PQ}}$ & $1417.8^{\mathrm{C}}$ \\
\hline $6.25 \mathrm{dsm}^{-1}$ & $1220.3^{Q}$ & $1224.0^{\mathrm{PQ}}$ & $1644.5^{\mathrm{L}-\mathrm{P}}$ & $1241.8^{\mathrm{PQ}}$ & $1572.5^{\mathrm{I}-\mathrm{N}}$ & $1457.7^{\mathrm{I}-\mathrm{L}}$ & $1359.8^{\mathrm{M}-\mathrm{Q}}$ & $1568.2^{\mathrm{I}-\mathrm{N}}$ & $1380.9^{\mathrm{I}-\mathrm{M}}$ & $1213.4^{\mathrm{K}-\mathrm{P}}$ & $1378.3^{\mathrm{C}}$ \\
\hline Mean B & $1270.8^{\mathrm{D}}$ & $1515.9^{\mathrm{C}}$ & $1848.2^{\mathrm{A}}$ & $1572.9^{\mathrm{DE}}$ & $1668.0^{\mathrm{C}}$ & $1598.8^{\mathrm{B}}$ & $1440.2^{\mathrm{C}}$ & $1516.4^{\mathrm{C}}$ & $1384.9^{\mathrm{D}}$ & $1272.7^{\mathrm{E}}$ & \\
\hline
\end{tabular}


Table 3.Effect of foliar application on oil percent (\%) of geranium under salt stress during seasons 2016 and 2017.

\begin{tabular}{|c|c|c|c|c|c|c|c|c|c|c|c|}
\hline \multirow{2}{*}{$\begin{array}{r}\text { Foliar } \\
\text { application }\end{array}$} & \multicolumn{3}{|c|}{ Moringa extracts $\left(g-L^{-1}\right)$} & \multicolumn{2}{|c|}{ Ascorbic acid(gL $\left(\mathrm{gL}^{-1}\right)$} & \multicolumn{3}{|c|}{ Proline acid $\left(\mathrm{mgL}^{-1}\right)$} & \multirow[b]{2}{*}{100} & \multirow[b]{2}{*}{ Control } & \multirow[b]{2}{*}{ Mean A } \\
\hline & 2.5 & 5 & 10 & 0.1 & 0.2 & 0.3 & 25 & 50 & & & \\
\hline Salinity & \multicolumn{11}{|c|}{ First season } \\
\hline Tap & $0.07^{\mathrm{NO}}$ & $0.06^{\mathrm{OP}}$ & $0.10^{\mathrm{LM}}$ & $0.06^{\mathrm{O}}$ & $0.07^{\mathrm{NO}}$ & $0.14^{\mathrm{PQ}}$ & $0.07^{\mathrm{NO}}$ & $0.06^{\mathrm{OP}}$ & $0.03^{Q}$ & $0.15^{\mathrm{GH}}$ & $0.08^{\mathrm{D}}$ \\
\hline $3.125 \mathrm{dsm}^{-1}$ & $0.14^{\mathrm{HI}}$ & $0.15^{\mathrm{GH}}$ & $0.15^{\mathrm{GH}}$ & $0.11^{\mathrm{J}-\mathrm{M}}$ & $0.11^{\mathrm{J}-\mathrm{M}}$ & $0.23^{\mathrm{DE}}$ & $0.23^{\mathrm{CDE}}$ & $0.18^{\mathrm{F}}$ & $0.13^{\mathrm{HIJ}}$ & $0.11^{\mathrm{J}-\mathrm{M}}$ & $0.15^{\mathrm{B}}$ \\
\hline $4.69 \mathrm{dsm}^{-1}$ & $0.18^{\mathrm{F}}$ & $0.11^{\mathrm{J}-\mathrm{M}}$ & $0.21^{\mathrm{E}}$ & $0.07^{\mathrm{NO}}$ & $0.11^{\mathrm{KLM}}$ & $0.21^{\mathrm{CD}}$ & $0.13^{\mathrm{HIJ}}$ & $0.13^{\mathrm{HIJ}}$ & $0.13^{\mathrm{HIJ}}$ & $0.11^{\mathrm{KLM}}$ & $0.14^{\mathrm{C}}$ \\
\hline $6.25 \mathrm{dsm}^{-1}$ & $0.12^{\mathrm{I}-\mathrm{L}}$ & $0.13^{\mathrm{HIJ}}$ & $0.40^{\mathrm{A}}$ & $0.07^{\mathrm{NO}}$ & $0.18^{\mathrm{FG}}$ & $0.25^{\mathrm{BC}}$ & $0.12^{\mathrm{IJK}}$ & $0.22^{\mathrm{DE}}$ & $0.27^{\mathrm{B}}$ & $0.09^{\mathrm{MN}}$ & $0.18^{\mathrm{A}}$ \\
\hline Mean B & $0.13^{\mathrm{D}}$ & $0.11^{\mathrm{E}}$ & $0.22^{\mathrm{A}}$ & $0.08^{\mathrm{F}}$ & $0.12^{\mathrm{E}}$ & $0.21^{\mathrm{B}}$ & $0.14^{\mathrm{CD}}$ & $0.15^{\mathrm{C}}$ & $0.14^{\mathrm{C}}$ & $0.12^{\mathrm{E}}$ & \\
\hline Salinity & \multicolumn{11}{|c|}{ Second season } \\
\hline Tap & $0.12^{\mathrm{N}-\mathrm{R}}$ & $0.10^{\mathrm{O}-\mathrm{R}}$ & $0.10^{\mathrm{O}-\mathrm{R}}$ & $0.08^{\mathrm{RS}}$ & $0.08^{\mathrm{RS}}$ & $0.06^{\mathrm{ST}}$ & $0.06^{\mathrm{ST}}$ & $0.04^{\mathrm{TU}}$ & $0.03^{\mathrm{U}}$ & $0.16^{\mathrm{IJK}}$ & $0.08^{\mathrm{D}}$ \\
\hline $3.125 \mathrm{dsm}^{-1}$ & $0.18^{\mathrm{GHI}}$ & $0.17^{\mathrm{HIJ}}$ & $0.25^{\mathrm{CD}}$ & $0.19^{\mathrm{GH}}$ & $0.13^{\mathrm{L}-\mathrm{O}}$ & $0.23^{\mathrm{DEF}}$ & $0.21^{\mathrm{EFG}}$ & $0.19^{\mathrm{GHI}}$ & $0.13^{\mathrm{K}-\mathrm{N}}$ & $0.11^{\mathrm{NQ}}$ & $0.18^{\mathrm{B}}$ \\
\hline $4.69 \mathrm{dsm}^{-1}$ & $0.23^{\mathrm{DEF}}$ & $0.11^{\mathrm{N}-\mathrm{R}}$ & $0.21^{\mathrm{FG}}$ & $0.09^{\mathrm{QRS}}$ & $0.11^{\mathrm{N}-\mathrm{Q}}$ & $0.27^{\mathrm{BC}}$ & $0.15^{\mathrm{JKL}}$ & $0.12^{\mathrm{M}-\mathrm{P}}$ & $0.15^{\mathrm{JKL}}$ & $0.12^{\mathrm{M}-\mathrm{P}}$ & $0.16^{\mathrm{C}}$ \\
\hline $6.25 \mathrm{dsm}^{-1}$ & $0.10^{\mathrm{O}-\mathrm{R}}$ & $0.11^{\mathrm{N}-\mathrm{Q}}$ & $0.36^{\mathrm{A}}$ & $0.09^{\mathrm{PQR}}$ & $0.24^{\mathrm{DE}}$ & $0.27^{\mathrm{BC}}$ & $0.15^{\mathrm{J}-\mathrm{M}}$ & $0.34^{\mathrm{A}}$ & $0.29^{\mathrm{B}}$ & $0.11^{\mathrm{N}-\mathrm{R}}$ & $0.21^{\mathrm{A}}$ \\
\hline Mean B & $0.16^{\mathrm{D}}$ & $0.12^{\mathrm{E}}$ & $0.23^{\mathrm{A}}$ & $0.11^{\mathrm{E}}$ & $0.14^{\mathrm{D}}$ & $0.21^{\mathrm{B}}$ & $0.14^{\mathrm{D}}$ & $0.17^{\mathrm{C}}$ & $0.15^{\mathrm{D}}$ & $0.13^{\mathrm{E}}$ & \\
\hline
\end{tabular}

Means within the letter and having the letters are not significantly from each other at significantly level of 0.05

Table 4. Effect of foliar application on oil yield per Hectare (L) of geranium under salt stress during seasons 2016 and 2017.

\begin{tabular}{|c|c|c|c|c|c|c|c|c|c|c|c|}
\hline \multirow{2}{*}{$\begin{array}{r}\text { Foliar } \\
\text { application }\end{array}$} & \multicolumn{2}{|c|}{ Moringa extracts $\left(\mathrm{gL}^{-1}\right)$} & \multicolumn{3}{|c|}{ Ascorbic acid(gL $\left(\mathrm{gL}^{-1}\right)$} & \multicolumn{3}{|c|}{ Proline acid $\left(\mathrm{mgL}^{-1}\right)$} & \multirow[b]{2}{*}{100} & \multicolumn{2}{|c|}{ Foliar applications } \\
\hline & 2.5 & 5 & 10 & 0.1 & 0.2 & 0.3 & 25 & 50 & & Control & Mean A \\
\hline & \multicolumn{11}{|c|}{ First season 2016} \\
\hline Tap & $3585.4^{\mathrm{DE}}$ & $3620.8^{\mathrm{DE}}$ & $4662.5^{\mathrm{A}}$ & $3407.8^{\mathrm{EF}}$ & $3622.1^{\mathrm{DE}}$ & $4496.9^{\mathrm{A}}$ & $3163.9^{\mathrm{AB}}$ & $3155.3^{\mathrm{BC}}$ & $3376.1^{\mathrm{FG}}$ & $1779.4^{\mathrm{Q}-\mathrm{U}}$ & $3487.0^{\mathrm{A}}$ \\
\hline $3.125 \mathrm{dsm}^{-1}$ & $2363.8^{\mathrm{K}-\mathrm{O}}$ & $2382.7^{\mathrm{KO}}$ & $3795.4^{\mathrm{CD}}$ & $2614.3^{\mathrm{IJK}}$ & $3311.5^{\mathrm{EF}}$ & $2604.9^{\mathrm{IJK}}$ & $1807.4^{\mathrm{Q}-\mathrm{T}}$ & $2112.7^{\mathrm{M}-\mathrm{Q}}$ & $3103.9^{\mathrm{FGH}}$ & $1681.7^{\mathrm{PQR}}$ & $2507.8^{\mathrm{B}}$ \\
\hline $4.69 \mathrm{dsm}^{-1}$ & $2069.5^{\mathrm{U}}$ & $2079.3^{\mathrm{TU}}$ & $3640.3^{\mathrm{BC}}$ & $2068.9^{\mathrm{NQ}}$ & $1571.3^{\mathrm{STU}}$ & $2531.50^{\mathrm{IL}}$ & $1458.5^{\mathrm{U}}$ & $2439.1^{\mathrm{JM}}$ & $2501.5^{\mathrm{EF}}$ & $1503.4^{\mathrm{TU}}$ & $2176.3^{\mathrm{D}}$ \\
\hline $6.25 \mathrm{dsm}^{-1}$ & $869.5^{\mathrm{U}}$ & $1485.5^{\mathrm{TU}}$ & $2440.3^{\mathrm{BC}}$ & $2039.7^{\mathrm{NQ}}$ & $1556.5^{\mathrm{STU}}$ & $2531.5^{\mathrm{IL}}$ & $1458.5^{\mathrm{U}}$ & $2438.2^{\mathrm{JM}}$ & $2160.3^{\mathrm{EF}}$ & $1503.4^{\mathrm{TU}}$ & $1848.3^{\mathrm{D}}$ \\
\hline Mean B & $2222.1^{\mathrm{E}}$ & $2392.1^{\mathrm{D}}$ & $3634.6^{\mathrm{A}}$ & $2532.7^{\mathrm{D}}$ & $2515.4^{\mathrm{C}}$ & $3041.1^{\mathrm{B}}$ & $1972.1^{\mathrm{D}}$ & $1926.6^{\mathrm{C}}$ & $2785.5^{\mathrm{B}}$ & $1616.9^{\mathrm{F}}$ & \\
\hline Salinity & \multicolumn{11}{|c|}{ Second season 2017} \\
\hline Tap & $2282.2^{\mathrm{E}-\mathrm{I}}$ & $2203.2^{\text {F-I }}$ & $3406.6^{\mathrm{A}}$ & $2905.6^{\mathrm{AD}}$ & $3228.7^{\mathrm{AB}}$ & $2129.0^{\mathrm{G}-\mathrm{J}}$ & $1935.8^{\mathrm{G}-\mathrm{J}}$ & $2508.7^{\mathrm{D}-\mathrm{G}}$ & $2675.7^{\mathrm{C}-\mathrm{F}}$ & $1283.0^{\mathrm{N}-\mathrm{Q}}$ & $2455.8^{\mathrm{A}}$ \\
\hline $3.125 \mathrm{dsm}^{-1}$ & $1401.4^{-Q}$ & $2067.8^{\mathrm{G}-\mathrm{J}}$ & $2203.2^{\mathrm{F}-\mathrm{I}}$ & $1479.5^{\mathrm{K}-\mathrm{P}}$ & $2338.9^{\mathrm{J}-\mathrm{O}}$ & $2479.9^{\mathrm{DG}}$ & $1891.4^{\mathrm{I}-\mathrm{L}}$ & $2124.7^{\mathrm{G}-\mathrm{J}}$ & $2213.8^{\mathrm{F}-\mathrm{I}}$ & $1042.8^{\mathrm{P}-\mathrm{S}}$ & $1854.3^{\mathrm{B}}$ \\
\hline $4.69 \mathrm{dsm}^{-1}$ & $1799.8^{\mathrm{S}}$ & $1448.6^{\mathrm{K}-\mathrm{Q}}$ & $2452.1^{\mathrm{D}-\mathrm{H}}$ & $2202.9^{\mathrm{F}-\mathrm{I}}$ & $2553.4^{\mathrm{D}-\mathrm{G}}$ & $2449.7^{\mathrm{DH}}$ & $1320.7^{\mathrm{M}-\mathrm{Q}}$ & $1218.5^{\mathrm{O}-\mathrm{R}}$ & $1831.7^{-\mathrm{M}}$ & $1117.2^{\mathrm{O}-\mathrm{R}}$ & $1839.5^{\mathrm{C}}$ \\
\hline $6.25 \mathrm{dsm}^{-1}$ & $658.8 .5^{\mathrm{S}}$ & $1420.2^{\mathrm{K}-\mathrm{P}}$ & $2368.7^{\mathrm{ABC}}$ & $1674.9^{\mathrm{RS}}$ & $1649.3^{\mathrm{QRS}}$ & $2448.7^{\mathrm{B}-\mathrm{E}}$ & $926.6^{\mathrm{QRS}}$ & $1761.8^{\mathrm{I}-\mathrm{N}}$ & $1313.7^{\mathrm{D} \_\mathrm{H}}$ & $1132.4^{\mathrm{MQ}}$ & $1525.5^{\mathrm{C}}$ \\
\hline Mean B & $1535.5^{\mathrm{G}}$ & $1784.9^{\mathrm{E}}$ & $2607.7^{\mathrm{A}}$ & $2065.7^{\mathrm{E}}$ & $2442.5^{\mathrm{E}}$ & $2376.8^{\mathrm{B}}$ & $1518.6^{\mathrm{F}}$ & $1903.4^{\mathrm{DE}}$ & $2008.7^{\mathrm{DE}}$ & $1143.9^{\mathrm{BC}}$ & \\
\hline
\end{tabular}


Table 5. Essential oil composition of Geranium plant.

\begin{tabular}{|c|c|c|c|c|}
\hline Component & $\begin{array}{l}\text { Moringa }\left(10 \mathrm{gL}^{-1}\right) \text { salt } \\
\left(6.25 \mathrm{dSm}^{-1}\right)\end{array}$ & $\begin{array}{l}\text { Tap } \\
\text { Salt (0) }\end{array}$ & $\begin{array}{l}\text { Proline }\left(25 \mathrm{mgL}^{-1}\right) \\
\text { Salt }\left(6.25 \mathrm{dSm}^{-1}\right)\end{array}$ & $\begin{array}{l}\text { Ascorbic acid (0.3 } \\
\left.\text { gL }^{-1}\right) \\
\text { Salt (0) }\end{array}$ \\
\hline Pinene & 0.55 & 1.47 & 1.03 & 0.65 \\
\hline Myrcene & 1.15 & 2.42 & 0.76 & 0.98 \\
\hline P-Cymene & 3.18 & 1.53 & 1.91 & 1.64 \\
\hline Isonenthone & 2.15 & 6.31 & 1.26 & 5.78 \\
\hline Linalool & 5.52 & 1.89 & 0.46 & 0.59 \\
\hline Citrenelylformate & 1.86 & 4.59 & 3.63 & 1.22 \\
\hline Cetronelol & 33.84 & 8.98 & 2.69 & 45.64 \\
\hline Geraniol & 19.74 & 3.01 & 1.68 & 10.71 \\
\hline Eugenol & 11.55 & 1.85 & 3.27 & 2.57 \\
\hline Geraniolbutrote & 1.94 & 3.76 & 1.90 & 1.43 \\
\hline B-Caryphyllene & 2.76 & 1.09 & 1.39 & 1.20 \\
\hline Unknown & 15.83 & 63.1 & 80.02 & 27.5 \\
\hline
\end{tabular}

Conclusion: We can conclude that, the geranium plant growth and productivity of essential oil decline sharply with increase irrigation water salinity. Using foliar application can reduce the hazards of high salinity level of irrigation water. Using moringa extract as foliar application led to improving plant growth and produced oil under different salinity level. Finally, it can be concluded that utilize in the foliar spray of moringa extracts to avoid the salt stress, following vitamin C which protects well cells and elongates the roots. The results were very useful for a farmer in the newly reclaimed areas who face limited saline irrigation water resources.

\section{REFERENCES}

Abdalla, M. M. (2013). The potential of Moringaoleifera extract as a biostimulant in enhancing the growth, biochemical and hormonal contents in rocket (Erucavesicaria subsp. sativa) plants. Int. J. Plant Physiol. Biochem., 5(3):42-49. DOI: 10.5897/IJPPB2012.026.

Andre, 'M., O. A. Bovi, N. B. Maia, L. E. S. Barata, R. D. C. Z. De Souza, E. M. R. De Souza, A. R.A. De Moraes and M. Q. Pinheiro (2008). Basil plants growth and essential oil yield in production system with successive cuts. Bragantia Campinas 67(2):385-389. Doi.org/10.1590/S0006-87052008000200014.

Aziz, E. E., H. Al-Amir, and L. E. Craker (2008): Influence of salt stress on growth and essential oil production in peppermint, pennyroyal and apple mint, J. Herbs, Spices and Medicinal plants, 14(1): 77-87. https://doi.org/10.1080/ 10496470802341375 .

Baâtour, O., M. B. Nasri-Ayachi, H. Mahmoudi, I. Tarchoun, N. Nassri, R. Kaddour, J. G.
Hamdaoui, B. Marzouk. and M. Lachaâl (2012). Essential oil yield and trachoma structure in two sweet marjoram (Origanummarjorana L.) varieties under salt stress. J. Med. Plants Res., 6: 3614-3623. DOI 10.5897/JMPR11.1619.

Biniecka, M. and S. Caroli (2011). Analytical methods for the quantification of volatile aromatic compounds. Trends in Analytical Chemistry, 30, No. 11. Elsevier Ltd. All rights reserved. Doi:10.1016/j.trac.2011.06.015.

Caliskan, O., D. Kurt, K. E. Temizel and M. S. Odabas (2017). Effect of salt stress and irrigation water on growth and development of sweet basil (Ocimumbasilicum L.). Open Agr. J.,2: 589594. Doi.org/10.1515/opag-2017-0062.

Cornillon, P. and A. Palliox (1997). Influence of sodium chloride on the growth and mineral nutrition of pepper cultivars. J. Plant Nutr., 20: 1085-1094. Doi.org/10.1080/01904169709365320.

Dadkhah A. R. (2010). Effect of salt stress on growth and essential oil of Matricaria chamomile. Rjbsci.,5(10):643-646. Doi. org/10.3923/rjbsci. 2010.643.646.

Eid, R., A., Taha, L. S. and Ibrahiem, S. M.M. (2011): Alleviation of adverse effects of salinity on growth, and chemical constituents of marigold plants by using glutathione and ascorbate. J. App. Sci. Res.,7(5): 714-721.Doi:10.1155/ 2012/217037 Shelden MC.

El-Baz, F. K., A. Amal, M. Amina and A. Aly (2003). Development of biochemical markers for salt stress tolerance in cucumber plants. Pakistan J. Biolog. Sci., 6: 16-22. DOI: 10.3923/pjbs. 2003.16.22.

Jelali, N., W. Dhifi, T. Chahed and B. Marzouk (2010). Salinity effects on growth, essential oil yield, and composition and phenolic compounds 
content of marjoram (origanummajorana L.) leaves. J. Food Biochem., 35:1443-1450. Doi:10.1111/j.1745-4514.2010.00465

Maia, S.S.S., da Silva, R.C.P., de Oliveira, F. de A., da Silva, O.M. dos P., da Silva, A.C. and Candido, W. dos S. (2017). Responses of basil cultivars to irrigation salinity. Rev. Brad. Eng. Agríc. Ambient., 21(1):44-49. Doi.org/10.1590/18071929/agriambi.v21n1 p44-49.

Mazher, A A. M., S. M. Zaghloul, S. A. Mahmoud and H. S. Siam (2011): Stimulatory effect of kinetin, ascorbic acid and glutamic acid on growth and chemical constituents of Codiaeum variegatum L. plants. American-Eurasian J. Agric. \& Environ. $\quad$ Sci., $10 \quad$ (3): 318-323. Doi.org/20113221915.

Soltani, Y., V. R. Saffari and A. A. M. Moud, (2014). Response of growth, flowering and some biochemical constituents of Calendula officinalis L. to foliar application of salicylic acid, ascorbic acid and thiamine. Ethno-
Pharmaceutical products, 1(1):37-44. Doi 10.1.1.896.8792

Veras, M. L. M., J. S. Filho, L. Alves, K. B. Silva, E. Franklin de Mesquita, R. Andrade, K. Belarmino, M.A. de Souza, Ribeiro, D. V., A. C. G. Neto, L. U. da Silva, J. Silva and A. S. F. Linhares (2017). Morpho-physiology of peppermint irrigated with salt water and bovine biofertilizer. Afr. J. Biotechnol. 16(23): 13141323.DOI: $10.5897 / \mathrm{AJB} 2016.15808$.

Waller, R. A. and D. B. Duncan (1969). A bays rule for the symmetric multiple comparison problems. Amer. Stat. Assoc. J. Dec. JASA, (64): 14841503.DOI: $10.1198 / 0003130031414$.

Weiss, E. A. (1997). Essential oil crops. New York: CAB (Centre for Agriculture and Biosciences). International. DOI 10.1007/978-90-481-2716$088, \mathrm{C}$.

Zoran P. Z., S. D J. Filip, S. S. Vidovic, D. S. Adamovic and M. Ahmed Elgandi (2015). Basil essential oil and extracts obtained by super critical fluid extraction. Original Scientific paper 46:259-269. .DOI: $\quad 10.2298 / \mathrm{APT} 1546259 \mathrm{Z}$. 